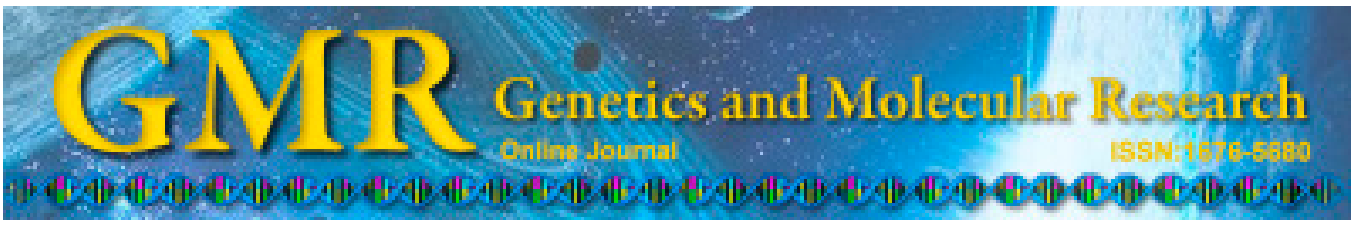

\title{
Effects of Astragaloside IV on diabetic nephropathy in rats
}

\author{
W.-S. Lu ${ }^{1,2 *}$, S. Li ${ }^{2 *}$, W.-W. Guo ${ }^{2 *}$ L.-L. Chen ${ }^{1,2}$ and Y.-S. Li ${ }^{1}$ \\ ${ }^{1}$ Medical College of Jinan University, Guanzhou, Guangdong Province, China \\ ${ }^{2}$ Shenzhen People's Hospital, Second Clinical Medical College of Jinan University, \\ Shenzhen, Guangdong Province, China \\ *These authors contributed equally to this study. \\ Corresponding author: Y.-S. Li \\ E-mail: syzyklys@126.com
}

Genet. Mol. Res. 14 (2): 5427-5434 (2015)

Received July 29, 2014

Accepted January 21, 2015

Published May 22, 2015

DOI http://dx.doi.org/10.4238/2015.May.22.12

ABSTRACT. The aims of this study were to explore the effects of Astragaloside IV on diabetic nephropathy (DN) rats. A total of 38 male Sprague-Dawley (SD) rats were divided into three groups: 10 in the normal (control) group, 14 in the DN model group, and 14 in the AS-IV group. Treatment began one week after the streptozotocin DN model was successfully established. Blood glucose and urine micro-albumin levels were measured every four weeks. After being treated for 12 weeks, all SD rats were sacrificed for blood and renal specimen collection. Renal cortex specimens were observed after hematoxylin and eosin and Masson staining. Expression levels of protein $\beta 1, \beta 1$-integrinlinked protein kinase (ILK) and $\alpha$-actinin- 4 were also measured. After eight weeks of intervention, blood glucose levels in the AS-IV group decreased significantly when compared with those of the model group $(\mathrm{P}<0.01)$. By the end of the twelfth week, the urine micro-albumin levels showed significant differences $(\mathrm{P}<0.01)$ between the AS-IV and model groups, and the expression levels of integrin $\beta 1$, ILK, and $\alpha$-actinin-4 also showed significant differences ( $\mathrm{P}<0.05$, respectively). Concomitantly, expression levels of integrin $\beta 1$, ILK, and $\alpha$-actinin- 4 
in the model group were significantly different from those of normal group $(\mathrm{P}<0.05)$. These results suggest that AS-IV can be quite effective in decreasing blood glucose levels, reducing urine albumin excretion, and improving the adhesion function of potocytes, and can thus delay the development of DN.

Key words: Astragaloside IV; Diabetic nephropathy; Potocyte; Albuminuria

\section{INTRODUCTION}

Research has shown that the loss of potocytes was positively correlated with urinary protein excretion in patients with diabetic nephropathy (DN) (Reidy and Susztak, 2009; Weil et al., 2012). Potocytes could be found in urine in 50\% of patients with type 2 diabetes and micro-albuminuria. For those who already had massive albuminuria, this rate rose up to $80 \%$ (Teiken et al., 2008). This suggested that abnormal adhesion, or even detachment of potocytes at the glomerular basement membrane, is one of the main factors resulting in DN albuminuria. Toyoda et al. (2007) found that some of the potocytes detected in urine were still active, indicating that detachment of potocytes, when compared with apoptosis, plays a more important role in the pathogenesis of DN albuminuria. The aim of the present study was to explore the therapeutic effect of astragaloside IV (AS-IV) on blood glucose, albuminuria, and the adhesion function of potocytes of patients with DN, and its underlying molecular mechanism.

\section{MATERIAL AND METHODS}

\section{Materials}

\section{Animals}

The study consisted of 38 specific pathogen-free male Sprague-Dawley (SD) rats at eight weeks old, weighing $200 \pm 20 \mathrm{~g}$, which were purchased from the Experimental Animal Center, Guangzhou University of Traditional Chinese Medicine (Qualified No. 0010232). After one week of adaptive breeding in the Experimental Animal Center, Medical College of Jinan University, rats were measured for their fasting blood glucose levels, which were all below $7 \mathrm{mM}$.

\section{Drugs}

Chemicals used in this study were as follows: AS-IV (98\% dry powder, Chengdu Jintaihe Pharmaceutical Chemical Technology Co., Ltd., China), streptozotocin (STZ, 98\% dry powder, Sigma-Aldrich, St. Louis, MO, USA), mouse anti-rat $\beta 1$-integrin-linked protein kinase (ILK) monoclonal antibody (sc-20019, Santa Cruz Biotechnology, Santa Cruz, CA, USA), mouse anti-rat $\alpha$-actinin-4 monoclonal antibody (sc-134236, Santa Cruz), mouse anti-rat integrin $\beta 1$ monoclonal antibody (sc-8978, Santa Cruz), two-step immunohistochemical detection kit (PV-9000, Zhongshan Golden Bridge Biotechnology Co., Beijing, China), DAB staining 
kit (Zhongshan Golden Bridge Co.), hematoxylin (Sigma-Aldrich), eosin Y, aniline blue, ponceau, acid fuchsin, and PAS staining kits (Nanjing Jiancheng Institute of Biological Engineering Co., Ltd., Nanjing, China).

\section{Instruments}

Equipment and instrumentation used in this study were as follows: blood glucose meter (Roche, Basel, Switzerland), 7020 automatic biochemical analyzer (Hitachi Co., Ltd., Products, Taiwan, China), optical microscope Eclipse 80i (Nikon Co., Tokyo, Japan), RM 2235 Rotary Slicer (Leica Co., Wetzlar, Germany), TB-718D biological tissue-embedding machine (Hubei Taiwei Medical Technology Co. Ltd., Hubei, China), $-80^{\circ} \mathrm{C}$ ultra-low temperature freezer (Thermo Fisher Scientific, Waltham, MA, USA), clean bench (Suzhou Antai Air Technology Co. Ltd., Suzhou, China).

\section{Methods}

\section{Grouping, model building, and medication}

SD rats were randomly divided into three groups: 10 in the normal group, 14 in the model group, and 14 in the AS-IV intervention group (38 total).

According to relevant literature (Zhang et al., 2012), after being fasted (water was provided ad libitum) for $12 \mathrm{~h}$, rats received an injection of streptozotocin in the left intraperitoneal cavity. After $72 \mathrm{~h}$, tail vein blood was collected for blood glucose measurements for the subsequent 3 days. Rats with three consecutive blood glucose measurements $>16.7 \mathrm{mM}$, with a urine volume more than $150 \%$ of the original amount, and with significantly increased water intake were confirmed as diabetic rats.

Rats in the intervention group received AS-IV $(40 \mathrm{mg} / \mathrm{kg})$ by gavage every day, while those in the normal and model groups were given the same amount of normal saline. No insulin or any other antidiabetic drugs were applied.

\section{Detection indices}

To determine the blood and urinary index, tail blood samples were tested with a glucose meter and biochemical analyzer; serum creatinine, urea nitrogen, urine protein and urinary creatinine were measured and the creatinine clearance rate (Ccr) was calculated. Blood glucose and $24 \mathrm{~h}$ urinary albumin were monitored dynamically.

For histopathological staining, hematoxylin and eosin (HE) and Masson staining was performed on the renal cortex to observe the condition of the glomerular mesangium and mesangial matrix; 10 glomeruli were chosen to calculate the area ratio, and mean values were taken for statistical analysis.

For immunohistochemical analysis, after pretreatment, the renal cortices were made into tissue sections and $\beta 1$ integrin, $\alpha$-actinin- 4 , and ILK were stained according to immunohistochemical methods. Ten glomeruli were randomly chosen for photography and measurement of the average integrated optical density (OD) values. The mean value of OD values in three images was used to reflect the intensity of immunohistochemical reactant (a kind of protein) expression in the tested rat. 


\section{Statistical analysis}

Quantitative data are reported as means \pm standard deviation (SD). Statistical analyses were conducted using the SPSS13.0 statistical software (SPSS, Chicago, IL, USA). One-way analysis of variance (ANOVA) was used to make comparisons among groups. Data obtained at different time points were analyzed by repeated-measures ANOVA. The least-squares difference test was applied in pairwise comparisons between groups. Data were considered to be statistically significant at $\mathrm{P}<0.05$.

\section{RESULTS}

\section{General condition of experimental rats}

Symptoms of hyperphagia, polydipsia, and polyuria were seen in rats of the model and intervention groups after injection of streptozotocin. During drug intervention, there were six deaths in the model group, four in the intervention group, and none in the normal control group.

\section{Improvement following AS-IV treatment in blood glucose and urinary albumin}

By the end of the eighth week, blood glucose levels of the AS-IV group were significantly lower than those of the model group $(\mathrm{P}<0.05)$. By the end of the twelfth week, microalbuminuria excretions of the AS-IV group decreased significantly when compared with the model group $(\mathrm{P}<0.01)$ (Tables 1 and 2 ).

Table 1. Glucose levels (mM) of the groups at different times [means \pm standard deviation (SD)].

\begin{tabular}{lrcccc}
\hline Group & $\mathrm{N}$ & 0 Week & 4th Week & 8th Week & 12 th Week \\
\hline Normal & 10 & $5.82 \pm 0.37$ & $5.56 \pm 0.42$ & $5.49 \pm 0.23$ & $5.52 \pm 0.15$ \\
Model & 8 & $24.39 \pm 1.83^{*}$ & $25.93 \pm 1.94^{*}$ & $26.49 \pm 1.34^{*}$ & $26.20 \pm 1.29 *$ \\
AS-IV & 10 & $24.92 \pm 1.73^{*}$ & $25.51 \pm 1.40^{*}$ & $21.21 \pm 2.23^{* *}$ & $16.29 \pm 2.03^{*}$ \\
\hline
\end{tabular}

$* \mathrm{P}<0.01$ vs normal group; ${ }^{\mathrm{P}}<0.01$ vs the model group. AS-IV $=$ astragaloside IV.

\begin{tabular}{|c|c|c|c|c|c|}
\hline & $\mathrm{N}$ & 0 Week & 4th Week & 8th Week & 12th Week \\
\hline Normal & 10 & $60.10 \pm 10.52^{\#}$ & $67.18 \pm 12.54^{\#}$ & $66.45 \pm 11.85^{\#}$ & $62.39 \pm 13.58^{\#}$ \\
\hline Model & 8 & $663.87 \pm 73.98^{*}$ & $1680.97 \pm 115.35^{*}$ & $1884.36 \pm 125.04 * \#$ & $2045.42 \pm 167.92^{* \#}$ \\
\hline AS-IV & 10 & $655.28 \pm 83.59 *$ & $1446.77 \pm 149.05^{* \#}$ & $1284.56 \pm 170.48^{* \#}$ & $932.61 \pm 142.85^{* \#}$ \\
\hline
\end{tabular}

$* \mathrm{P}<0.01$ vs the normal group; ${ }^{\text {P }}<0.01$ vs the model group.AS-IV $=$ astragaloside IV.

\section{Effects of astragaloside and astragalus polysaccharide on kidney histopathology}

In the model group, there was apparent proliferation in inherent components of the glomeruli such as in mesangial cells and mesangial matrix, as well as notable thickening of the basement membrane. The area ratio was significantly different from that of the normal group and the AS-IV group $(\mathrm{P}<0.01)$ (Figure 1A-F; Table 3). 


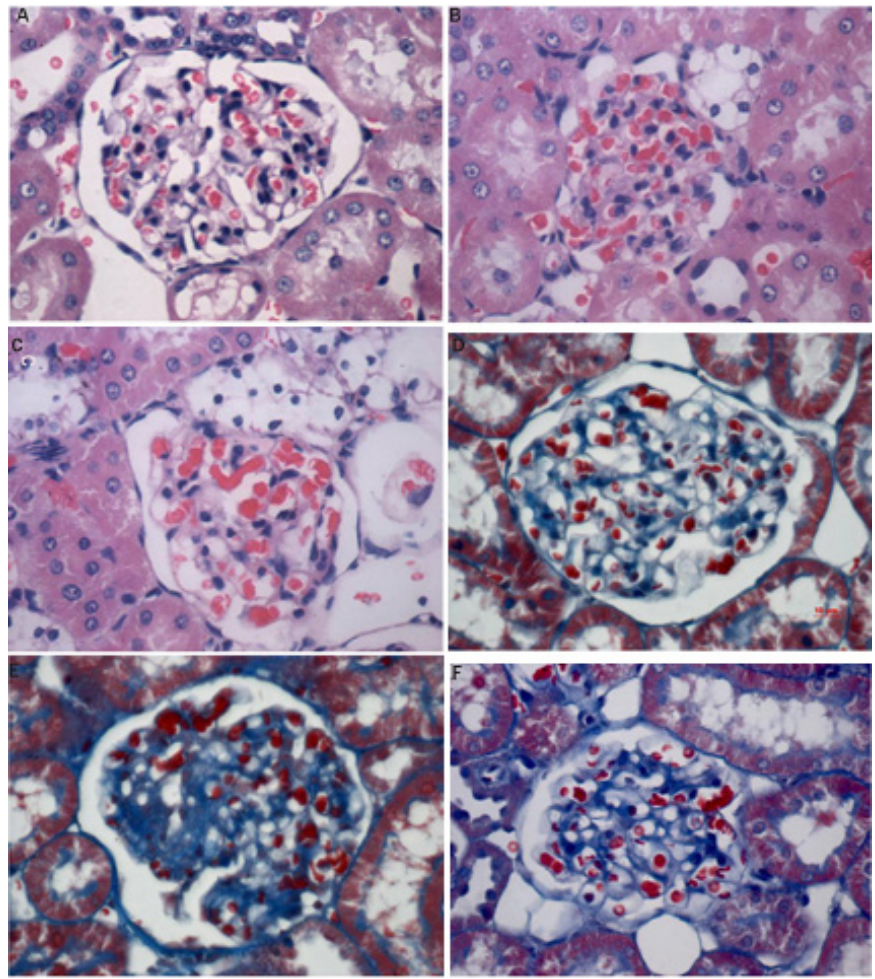

Figure 1. Pathological observation of AS-IV on renal function in diabetic nephropathy (400X). A) Normal group [hematoxylin and eosin (HE), 400X]; B) model group (HE, 400X); C) astragaloside-IV (AS-IV) group (HE, 400X); D) normal group (Masson, 400X); E) model group (Masson, 400X) F) AS-IV group (Masson, 400X).

Table 3. Proportion of glomerular basement membrane and glomerular intrinsic composition (SIC) in the different groups [means \pm standard deviation $(\mathrm{SD})]$.

\begin{tabular}{lccc}
\hline Group & N & SGBM (\%) & SIC (\%) \\
\hline Normal & 10 & $13.57 \pm 3.74^{*}$ & $20.30 \pm 5.95^{*}$ \\
Model & 8 & $21.66 \pm 4.89$ & $39.30 \pm 11.14$ \\
AS-IV & 10 & $19.65 \pm 4.66^{*}$ & $23.41 \pm 5.33^{*}$ \\
\hline
\end{tabular}

$* \mathrm{P}<0.01$ vs the model group. GBM = glomerular basement membrane; AS-IV = astragaloside IV.

\section{Effects of astragaloside on adhesion molecules and cytoskeletal proteins of poto- cytes in DN rats}

Compared with the intervention and normal groups, expression levels of $\beta 1$ integrin were significantly lower in the model group $(\mathrm{P}<0.05)$. Furthermore, when compared with the model group, expression levels of $\beta 1$ integrin were significantly higher in the model group ( $\mathrm{P}$ $<0.05)$. In model and AS-IV groups, expression levels of ILK and $\alpha$-actinin-4 were higher than in the normal group; this difference was statistically significant $(\mathrm{P}<0.01)$. Furthermore, the expression levels of ILK and $\alpha$-actinin-4 in the AS-IV group decreased significantly compared to those in the model group $(\mathrm{P}<0.01)$ (Figure 2A-F; Table 4). 


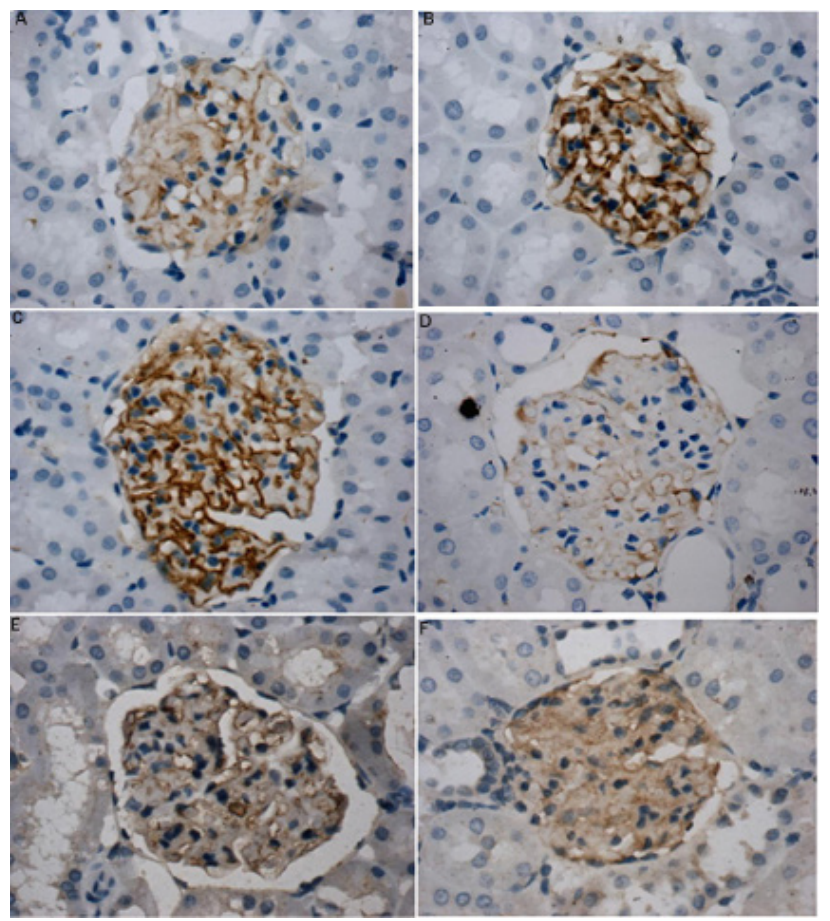

Figure 2. Immunohistochemical staining of $\alpha$-actin- 4 and $\beta 1$-integrin-linked protein kinase (ILK) in the potocytes of rats (400X). A) Normal group ( $\alpha$-actinin 4, 400X); B) astragaloside-IV (AS-IV) group ( $\alpha$-actinin-4, 400X); C) model group ( $\alpha$-actinin-4, 400X); D) normal group (ILK, 400X); E) AS-IV group (ILK, 400X); F) model group (ILK, 400X).

Table 4. Expression level of $\beta 1$ integrin, ILK, and $\alpha$-actinin-4 in renal tissue from the different groups examined by immunohistochemistry [means \pm standard deviation (SD)].

\begin{tabular}{lcccc}
\hline Group & $\mathrm{N}$ & $\beta 1$ integrin $(\mathrm{OD})$ & ILK $(\mathrm{OD})$ & $\alpha$-actinin-4 (OD) \\
\hline Normal & 10 & $117.06 \pm 14.49$ & $99.93 \pm 11.70$ & $164.05 \pm 18.19$ \\
Model & 8 & $80.08 \pm 11.61^{*}$ & $153.03 \pm 19.55^{* *}$ & $212.98 \pm 6.31^{* *}$ \\
AS-IV & 10 & $98.99 \pm 22.24^{* *}$ & $130.21 \pm 21.00^{* * * \#}$ & $189.13 \pm 19.28^{* * * \#}$ \\
\hline
\end{tabular}

*P $<0.05$ vs the normal group; ${ }^{*} \mathrm{P}<0.05$ vs the model group; $* * \mathrm{P}<0.01$ vs the normal group; ${ }^{\#} \mathrm{P}<0.01$ vs the model group. AS-IV = astragaloside IV; ILK = integrin-linked protein kinase.

\section{DISCUSSION}

As one of the primary microvascular complications of diabetes, $\mathrm{DN}$ is a common disease in the elderly, which can lead to end-stage renal failure. During early stages, no obvious imaging changes can be found in diabetic patients. However, as the disease progresses, hypertrophy of the kidneys occur, showing hyperplasia and hypertrophy of kidney cells in pathological anatomy. In DN, cell proliferation rates differ for all types of cells, causing different pathological changes in different locations. For example, in the early stages, hyperplasia mainly occurs in mesangial cells and tubular epithelial cells, whereas hypertrophy mainly occurs in the thickening of the basement membrane. In our study, biopsy results of the model and 
intervention groups were in complete accord with the above procession. Conventional concepts hold that hypertrophy of mesangial cells, increase of mesangial matrix, thickening of basement membrane, and accumulation of extracellular matrix is the primary mechanisms of proteinuria (Wolf and Ziyadeh, 1999). Being the main clinical characteristic, proteinuria is also one of the crucial factors of progressive deterioration of renal function. It usually starts from micro-albuminuria and gradually develops into heavy proteinuria (also known as nonselective proteinuria), and eventually leads to glomerular sclerosis and end-stage renal disease (Fukuda et al., 2012). From our study, it could also be seen that variations in mesangial cells, mesangial matrix, and the basement membrane play a crucial role in the development of proteinuria.

Potocytes are a type of highly differentiated epithelial cell located on the glomerular filtration membrane. The appropriate amount of potocytes and their integrity are crucial in maintaining regular permeability and function of the glomerular filtration membrane. With the improvement in recent knowledge of potocytes, it has now been accepted that damage to potocytes is the key to impairment of glomerular filtration barrier function and the development of proteinuria, and especially of micro-albuminuria during the early stages of DN.

Specific manifestations of potocyte damage include morphological damage, adhesive function impairment, and cell apoptosis, leading to cell detachment and reduction, causing impairment of filtration membrane integrity. Some studies (Reidy and Susztak, 2009; Weil et al., 2012) have reported a correlation between proteinuria development in patients with DN and the number and density of their potocytes, concluding that there exists a positive correlation between potocyte detachment ratio and the amount of urinary protein. Together, this information suggests abnormal adhesion and detachment of potocytes at the glomerular basement membrane to be one of the primary factors of proteinuria development in patients with DN.

The Chinese medicine Astragalus is the root of the legumous herb Mongolia Astragalus and Astragalus membranaceus. It has been applied for more than 2000 years in Chinese traditional medicine as the cure for syndromes of solid qi deficiency and qi stagnation. However, modern pharmacological studies have discovered its effect in immune-enhancement, liver protection, diuresis, anti-aging, anti-stress, blood pressure control, blood sugar control, and anti-bacterial activity.

The main effective components in Astragalus are astragaloside and astragalus polysaccharide. Recently, increasing numbers of studies have been targeted on astragalus and its effective components as potential intervention agents for diabetes and DN. Most results support its effect in diabetes mellitus and DN control during the mid-early phase. Our study also was in agreement with the favorable effects of astragalus on early DN, which include: 1) lowering blood sugar and improving high blood sugar condition; 2) extenuating renal hypertrophy and improving the state of microscopic pathology structure; 3) reducing 24-h urinary albumin thus reducing the loss of proteins and other macromolecules; 4) increasing the expression of cell adhesion molecules on potocytes and reducing potocyte detachment. Our study illustrated that AS-IV could increase the expression level of $\beta 1$ integrin in the rat renal cortex while decreasing that of $\alpha$-actinin- 4 and ILK. Therefore, it might be conjectured that an impact of ASIV on adhesion molecules is one of the reasons for its effect on blood sugar control, immune function regulation, and on its influence over a variety of pathological mechanisms in diabetes mellitus. In the theory of Chinese traditional medicine, as one of the major components in Astragalus, astragaloside probably has an effect of benefiting qi for astringency, and can thus improve the qi deficiency state of the human body, through maintenance of the integrity of potocyte structure and promoting the expression of adhesion molecules, so as to improve the vitality and adhesion function of the potocytes. 


\section{ACKNOWLEDGMENTS}

Research supported by Shenzhen Technology Research and Development Funded Projects (\#JCYJ201208030163410835).

\section{REFERENCES}

Fukuda A, Chowdhury MA, Venkatareddy MP, Wang SQ, et al. (2012). Growth-dependent podocyte failure causes glomerulosclerosis. J. Am. Soc. Nephrol. 23: 1351-1363.

Reidy K and Susztak K (2009). Epithelial-mesenchymal transition and podocyte loss in diabetic kidney disease. Am. J. Kidney Dis. 54: 590-593.

Teiken JM, Audettey JL, Laturnus DI, Zheng S, et al. (2008). Podocyte loss in aging OVE26 diabetic mice. Anat. Rec. (Hoboken) 291: 114-121.

Toyoda M, Najafian B, Kim Y, Caramori ML, et al. (2007). Podocyte detachment and reduced glomerular capillary endothelial fenestration in human type 1 diabetic nephropathy. Diabetes 56: 2155-2160.

Weil EJ, Lemley KV, Mason CC, Yee B, et al. (2012). Podocyte detachment and reduced glomerular capillary endothelial fenestration promote kidney disease in type 2 diabetic nephropathy. Kidney Int. 82: 1010-1017.

Wolf G and Ziyadeh FN (1999). Molecular mechanisms of diabetic renal hypertrophy. Kidney Int. 56: 393-405.

Zhang M, Liu M, Xiong M, Gong J, et al. (2012). Schisandra chinensis fruit extract attenuates albuminuria and protects podocyte integrity in a mouse model of streptozotocin-induced diabetic nephropathy. J. Ethnopharmacol. 141: 111-118. 